\title{
SYNTHESIS AND APPLICATIONS OF LATTICE IMAGE OPERATORS BASED ON FUZZY NORMS
}

\author{
Petros Maragos, Vassilis Tzouvaras, and Giorgos Stamou \\ National Technical University of Athens, \\ Dept. Electrical \& Computer Engineering, \\ Zografou 15773, Athens, Greece. \\ Email: maragos@cs.ntua.gr, tzouvaraseimage.ntua.gr
}

\begin{abstract}
In this paper we use concepts from the lattice-based theory of morphological operators and fuzzy sets to develop generalized lattice image operators that can be expressed as nonlinear convolutions that are suprema or infima of fuzzy intersection or union norms. Our emphasis (and differences with previous works) is the construction of pairs of fuzzy dilation and erosion operators that form lattice adjunctions. This guarantees that their composition will be a valid algebraic opening or closing. The power but also the difficulty in applying these fuzzy operators to image analysis is the large variety of fuzzy norms and the absence of systematic ways in selecting them. Towards this goal, we have performed extensive experiments in applying these fuzzy operators to various nonlinear filtering and image analysis tasks, attempting first to understand the effect that the type of fuzzy norm and the shape-size of structuring function have on the resulting new image operators. Further, we have developed some new fuzzy edge gradients and optimized their usage for edge detection on test problems via a parametric fuzzy norm.
\end{abstract}

\section{INTRODUCTION}

Mathematical morphology (MM) and fuzzy sets share many common theoretical concepts. As an earlier example, the use of min/max to extend the intersection/union of ordinary (crisp) sets to fuzzy sets [12] has also been used to extend the set-theoretic morphological shrink/expand operations on binary images to $\mathrm{min} / \mathrm{max}$ filtering on graylevel images [9, 3]. While the field of morphological image analysis was maturing, several researchers developed various other approaches in using fuzzy logic ideas for extending or generalizing the morphological image operations $[11,1]$. The main ingredients of these approaches have been to (1) map the max-plus structure of signal dilation to a sup- $T$ signal convolution, where $T$ is some fuzzy intersection norm, and (2) use duality to map the inf-plus structure of signal erosion to a inf- $T^{\prime}$ convolution, where $T^{\prime}$ is a dual fuzzy union norm. The main disadvantage of these approaches is that composition of the operators from steps (1) and (2) is not guaranteed to be an algebraic opening or closing. (Openings and closing are the basic morphological smoothing filters.) Another difficulty in applying fuzzy-based operators to various image analysis problems is the huge variety of fuzzy norms and the absence of

This research work was supported by the Greek Secretariat for Research and Technology and by the European Union under the program חENE $\Delta-$ 99 with Grant \# 99E $\Delta 164$. It was also partially supported by the ICCSNTUA basic research program 'Archimedes'. some practical experience or systematic ways in selecting the best of them for certain image applications.

Meanwhile MM was extended using lattice theory $[10,4]$ to more general operators that shared with the standard dilation, erosion, opening and closing only a few algebraic properties. One such fundamental algebraic structure is a pair of erosion/dilation operators that form an adjunction. This guarantees the formation of openings and closings.

In a previous work $[7,8]$ some of us used lattice theory to develop generalizations of morphological signal/vector operations for based on fuzzy norms. These operations were used in fuzzy dynamical systems to represent the mapping between input and ouput signals (via nonlinear fuzzy-based convolutions) and the mapping between state vectors (via generalized fuzzy-based products of matrices and vectors) as a generalized dilation or erosion acting on signal or vector lattices. In this paper we continue this work and apply our general theoretical results from [7] to developing useful nonlinear image operators for image analysis. (A work similar to our theoretical analysis appeared recently in [2].) First, we discuss the theoretical development of the new operators. Then, we present our results from extensive experiments in applying these fuzzy operators to various nonlinear filtering and detection tasks applied to soilsection images. The emhasis in our experimental work is to understand the effect that the algebraic type of fuzzy norm and the shape and size of the structuring function have on the resulting new image operators. Finally, we present some new fuzzy edge gradients and show how to optimize a parametric fuzzy norm used in a $1 \mathrm{D}$ edge detection problem.

\section{LATTICE OPERATORS USING FUZZY NORMS}

A poset $\mathcal{L}$ is any set equipped with a partial ordering $\leq$. The supremum $(\bigvee)$ and infimum $(\bigwedge)$ of any subset of $\mathcal{L}$ is its lowest upper bound and greatest lower bound, respectively, induced by the partial order; both are unique if they exist. The algebra $(\mathcal{L}, \vee, \wedge)$ is called a complete lattice if the supremum and infimum of any (finite or infinite) collection of its elements exists. An operator $\psi$ on a complete lattice $\mathcal{L}$ is called: increasing if it preserves the partial ordering $[F \leq G \Longrightarrow \psi(F) \leq \psi(G)]$; idempotent if $\psi^{2}=\psi$; antiextensive (extensive) if $\psi(F) \leq F(F \leq \psi(F))$. An operator $\varepsilon(\delta)$ on a complete semilattice is called an erosion (dilation) if it distributes over the infimum (supremum) of any collection of lattice elements; namely $\delta\left(\bigvee_{i} F_{i}\right)=\bigvee_{i} \delta\left(F_{i}\right)$ and $\varepsilon\left(\bigwedge_{i} F_{i}\right)=\bigwedge_{i} \varepsilon\left(F_{i}\right)$. An operator is called an opening (closing) if it is increasing, antiextensive (extensive) and idempotent. An 
operator pair $(\varepsilon, \delta)$ is called an adjunction iff $\delta(F) \leq G \Longleftrightarrow$ $F \leq \varepsilon(G), \forall F, G \in \mathcal{L}$.

Given a dilation $\delta$, there is a unique erosion $\varepsilon(G)=\bigvee\{F$ : $\delta(F) \leq G\}$ such that $(\varepsilon, \delta)$ is adjunction, and vice-versa. Given an adjunction $(\varepsilon, \delta)$, it can be shown [4] that: (i) $\delta$ is a dilation and $\varepsilon$ is an erosion. (ii) $\delta \varepsilon$ is an opening, and $\varepsilon \delta$ is a closing.

In this paper, the signal space is the collection $\mathcal{L}=\mathbb{V}^{\mathbb{E}}$ of all images/signals $F: \mathbb{E} \rightarrow \mathbb{V}$, where $\mathbb{E}=\mathbb{R}^{d}$ or $\mathbb{Z}^{d}, d=1,2, \ldots$, and $\mathbb{V}=[0,1]$. The value set $\mathbb{V}$, equipped with the usual ordering $\leq$ becomes a complete lattice. The signal space $\mathcal{L}$ also becomes a complete distributive lattice if we define on it the standard pointwise partial ordering $\leq$, supremum $\vee$, and infimum $\wedge$ induced by $\mathbb{V}$. It has been shown [4] that a pair $(\varepsilon, \delta)$ is an adjunction on the image lattice $\mathbb{V}^{\mathbb{E}}$ iff for every $x, y \in \mathbb{E}$ there exists a scalar adjunction $\left(\varepsilon_{x, y}, \delta_{x, y}\right)$ on $\mathbb{V}$ such that

$$
\delta(F)(y)=\bigvee_{x \in \mathbb{E}} \delta_{x, y}(F(x)), \quad \varepsilon(G)(x)=\bigwedge_{y \in \mathbb{E}} \varepsilon_{y, x}(G(y))
$$

In this paper we build generalized image dilations and erosions by defining the scalar dilations (erosions) $\delta_{x, y}\left(\varepsilon_{x, y}\right)$ via some fuzzy intersection (union) between the values of the image signal $F$ and a structuring function $H$. (More details can be found in [7].) First we define fuzzy norms.

A fuzzy intersection norm, in short a $T$ norm, is a binary operation $T:[0,1] \rightarrow[0,1]$ that satisfies the following conditions [6]: For all $a, b, c \in[0,1]$

F1. $T(a, 1)=a$ and $T(a, 0)=0$ (boundary conditions).

F2. $T(a, T(b, c))=T(T(a, b), c)$ (associativity).

F3. $T(a, b)=T(b, a)$ (commutativity).

F4. $b \leq c \Longrightarrow T(a, b) \leq T(a, c)$ (increasing).

For the $T$ norm to be a scalar dilation (with respect to any argument) on $\mathbb{V}$, it must also satisfy:

F5. $T$ is a continuous function.

A fuzzy union norm [6] is a binary operation $U:[0,1] \rightarrow[0,1]$ that satisfies F2-F5 and a dual boundary condition:

$\mathrm{F}^{\prime} . U(a, 0)=a$ and $U(a, 1)=1$.

Clearly, $U$ is an erosion on $\mathbb{V}$.

By defining in (1) the scalar dilations $\delta_{x, y}$ via a fuzzy Tnorm between $F$ and $H$ values [7], we can define a generalized image dilation and erosion by

$$
\begin{aligned}
& \delta(F)(y) \quad:=\bigvee_{x \in \mathbb{E}} T(F(x), H(y-x)), \\
& \varepsilon(G)(x):=\bigwedge_{y \in \mathbb{E}} \Xi(G(y), H(y-x))
\end{aligned}
$$

where $\Xi$ represents the adjoint scalar erosions $\left(\varepsilon_{x, y}\right)$ in (1) and is actually the adjoint of the fuzzy $T$ norm:

$$
T(v, a) \leq w \Longleftrightarrow v \leq \Xi(w, a)
$$

Given $T$ we can find its adjoint function $\Xi$ by

$$
\Xi(w, a)=\sup \{v \in[0,1]: T(v, a) \leq w\}
$$

Three examples of Tnorms are:

$$
\begin{aligned}
& \text { Min : } T_{1}(v, a)=\min (v, a) \\
& \text { Product : } T_{2}(v, a)=v \cdot a \\
& \text { Yager : } T_{3}(v, a)=1-\left(1 \wedge\left[(1-v)^{p}+(1-a)^{p}\right]^{1 / p}\right), p>0 .
\end{aligned}
$$

The corresponding three adjoint functions are:

$$
\begin{aligned}
& \Xi_{1}(w, a)= \begin{cases}w, & w<a \\
1, & w \geq a\end{cases} \\
& \Xi_{2}(w, a)= \begin{cases}\min (w / a, 1), & a>0 \\
1, & a=0\end{cases} \\
& \Xi_{3}(w, a)= \begin{cases}1-\left[(1-w)^{p}-(1-a)^{p}\right]^{1 / p}, & w<a \\
1, & w \geq a\end{cases}
\end{aligned}
$$

The generalized fuzzy opening $\alpha$ and closing $\beta$ are

$$
\alpha(F):=\delta(\varepsilon(F)), \quad \beta(F):=\varepsilon(\delta(F))
$$

If we define an alternative erosion operator by

$$
\varepsilon^{\prime}(F)(y)=\bigwedge_{x} U(F(x), H(y-x))
$$

where $U(a, b)=1-T(1-a, 1-b)$ is a fuzzy union that is dual to $T$, then $\varepsilon^{\prime}(F)=1-\delta(1-F)$; i.e., this second erosion $\varepsilon^{\prime}$ is the dual of the first dilation $\delta$. Further, the adjoint dilation $\delta^{\prime}$ of $\varepsilon^{\prime}$ is an operator that is dual of the first erosion $\varepsilon$. Previous works used pairs $\left(\varepsilon^{\prime}, \delta\right)$ or $\left(\varepsilon, \delta^{\prime}\right)$ which are duality pairs but not adjunctions and hence cannot form openings/closings via compositions.

\section{EXPERIMENTS AND EDGE GRADIENTS}

In this section we present some experimental results illustrating the differences between the classical morphological operators and the generalized operators based on fuzzy Tnorms, emphasizing the advantages of the parametric Tnorms. Further, we present some new edge gradients based on fuzzy operators and focus on the problem of optimally designing these fuzzy operators.

The first part of the experiments (Rows 1,2) deals with 1D images. We apply several fuzzy lattice operators based on $T$ norms and we compare the outcomes with the morphological operators. Rows 1 and 2 of Fig. 1 show the outputs of dilation, erosion, opening and closing operators, first for the morphological type using a 51pixel flat structuring element and second for the fuzzy type (2), (3) and (6) using a parabolic non-flat structuring function

$$
H[n]= \begin{cases}1-k(n / s)^{2}, & |n| \leq s \\ 0, & |n|>s\end{cases}
$$

with $s=25$. The parameter $s$ determines the scale, while $k$ affects the shape of $H$. (We used $k=0.5$.) We have experimented with three Tnorms: the minimum, the product and the Yager. The paramer $p$ of the Yager $T$ norm was $p=2$. For $p \downarrow 0$ the Yager $T$ norm takes the form of the drastic Tnorm, which is one extreme of the spectrum of $T$ norms, whereas for $p \rightarrow \infty$ it coincides with the minimum $T$ norm which is the other extreme of the spectrum. In general, we have observed that, the fuzzy operators are more adaptive and track closer the peaks/valleys of the signal than the corresponding flat morphological operators of the same scale.

The second part of the experiments (Rows 3,4) deals with 2D images. For all fuzzy operators used in Rows 3,4 we used the Yager Tnorm with parameter $p=10$ and a conical structuring function $H$. In Row 3, Fig. 1(i) shows an original soilsection image and its dilation and erosion first of the morphological type in Figs.1(j,k) and then of the fuzzy type in Figs. 1(1,m). In both cases the structuring element had a $7 \times 7$-pixel support, being flat in the morphological case and conical in the fuzzy case. Figure 1(n) in 
Row 4 shows the corresponding fuzzy clos-opening (a smoothing filter).

The rest of the images in Row 4 deal with edge detection: Figue 1(o) shows a nonlinear edge-enhancing gradient of the type $\delta(F)-\varepsilon(F)$, where $\delta$ and $\varepsilon$ are the morphological dilation and erosion, respectively, of $f$ by $3 \times 3$-pixel flat structuring element. Figure 1(p) shows the same type of gradient but uses fuzzy dilation and erosion with a $3 \times 3$-pixel structuring function. In the continuous case (images defined on $\mathbb{R}^{2}$ ), if $\delta_{s}(F)=F \oplus s B$ and $\varepsilon_{s}(F)=F \ominus s B$ are the flat dilation and erosion of $F$ by multiscale disks $s B$, it is well-known that

$$
\lim _{s \downarrow 0}\left[\delta_{s}(F)(x)-\varepsilon_{s}(F)(x)\right] /(2 s)=\|\nabla F(x)\|
$$

for a differentiable function $F$. We have proven that the same result holds if we replace the morphological dilation with a multiscale fuzzy dilation

$$
\delta_{s}(F)(x)=\bigvee_{\|y\| \leq s} T(F(x-y), H(y / s))
$$

and similarly for the adjoint multiscale fuzzy erosion $\varepsilon_{s}$. For the edge gradients, the unit-scale structuring function $H(x),|x| \leq 1$, must be a unimodal symmetric structuring function with a global maximum $H(0)=1$

Figure 1(q) shows a different type of edge gradient:

$$
\text { FuzzyEdge }_{\min }(F)=\min \left[\delta_{s}(F), 1-\varepsilon_{s}(F)\right]
$$

where $\delta_{s}$ and $\varepsilon_{s}$ are the same fuzzy dilation and erosion as in Fig. 1(p) with $s=1$. Figure 1(r) shows a similar type of fuzzy edge gradient as in Fig. 1(q) but the min is replaced with max. The last two types of edge gradients were inspired by the standard discrete morphological gradient $\delta(F)-\varepsilon(F)$, but to make the gradient operator more consistent with fuzzy set theory we replaced the difference between dilation and erosion with min (or max) of the dilation and the fuzzy complement of the erosion. As shown in Row 4 of Fig. 1, these new fuzzy gradient operators have a quite promising behavior since they yield cleaner and sharper edge peaks than the morphological gradient.

In general, having a parameter in the Tnorm offers flexibility for the fuzzy operator that use it. By tuning this parameter we can find the optimum solution depending on the type of application, such as edge detection in the presence of noise or other feature detection in images with low contrast. The scale (support size) and the shape of the structuring function are also important factors, which can influence the behavior of the fuzzy operators. For a 1D edge detection test problem (see Row 5 of Fig. 1), we have explored the efficacy of the fuzzy gradients by varying two parameters, the parameter $p$ of the Yager $T$ norm and the parameter $s$, which is the scale of the parabolic structuring function (8) used. Figure 1(t) shows the difference-based fuzzy gradient $(p=10, s=5)$ of the $1 \mathrm{D}$ test signal in Fig 1(q). By varying $p$ and $s$, on can optimize the edge detection performance as measured by the peak height of the fuzzy derivative. Thus, the 3D graph in Fig. 1(u) shows the peak heights of the difference-based fuzzy gradient of the 1D test signal in Fig 1(q) for various values of $p \in[1,22]$ and $s \in[1,25]$. Clearly, for each value of $p$ there is an optimum value of $s$; e.g., for $p=6$ the optimum was $s=10$. There are many optimal solution pairs $(p, s)$, and this illustrates the advantages of having a parametric Tnorm. Finally, Fig. 1(v) shows the min-based fuzzy gradient (11) of the 1D test signal; clearly, this yields a sharper peak.
Concluding, the power but also the difficulty in applying the lattice-based fuzzy operators to image analysis is the huge variety of fuzzy norms and the absence of some practical experience or systematic ways in selecting them. In our on-going work we are investigating rigorous methods to design these new operators for various nonlinear filtering and image analysis tasks, such as edge and peak/valley detection, by optimizing the parametric fuzzy norms and structuring functions used. In parallel, by experimenting with the type of fuzzy norm and the shape-size of the structuring function, it is possible to adapt the new fuzzy operators so that their performance has many promising aspects compared with the standard morphological operators. Finally, by combining lattice-based MM and fuzzy set theory, we can create new operators, like the fuzzy edge gradients, that extend and improve the capabilities of the standard morphological operators.

\section{REFERENCES}

[1] I. Bloch and H. Maitre, "Fuzzy mathematical morphologies: a comparative study" Pattern Recognition 28, vol. 9, pp. 13411387, 1995.

[2] T. Q. Deng and H.J.A.M. Heijmans, "Grey-Scale Morphology Based on Fuzzy Logic", Technical Report PNA-R0012, CWI, Amsterdam, Dec.2000.

[3] V. Goetcherian, "From binary to greytone image processing using fuzzy logic concepts”, Pattern Recognition 12, pp.7-15, 1980.

[4] H. J. A. M. Heijmans, Morphological Image Operators, Academic Press, Boston, 1994.

[5] E. Kerre and M. Nachtegael, "Fuzzy Techniques in Image Processing: Techniques and Applications", in Studies in Fuzziness and Soft Computing, Vol. 52, Physica Verlag, 2000.

[6] G. J. Klir and B. Yuan, Fuzzy Sets and Fuzzy Logic: Theory and Applications, Prentice-Hall, 1995.

[7] P. Maragos, G. Stamou and S. Tzafestas, "A Lattice Control Model of Fuzzy Dynamical Systems in State-Space", in Mathematical Morphology and Its Application to Image and Signal Processing, J. Goutsias, L. Vincent and D. Bloomberg, Eds, Kluwer Acad. Publ., Boston, 2000, pp. 61-70.

[8] P. Maragos and S. Tzafestas, "A Lattice Calculus Unification of Min-Max Control Systems of the Morphological and Fuzzy Type", Proc. Int'l Symp. on Soft Computing in Engineering Applications (SOFTCOM-98), Athens, pp. 247-248, June 1998.

[9] Y. Nakagawa and A. Rosenfeld, "A note on the use of local min and max operations in digital picture processing", IEEE Trans. Syst., Man, Cybern., SMC-8, p.632-635, Aug.1978.

[10] J. Serra Ed., Image Analysis and Mathematical Morphology II: Theoretical Advances, Acad. Press, NY, 1988.

[11] D. Sinha and E. R. Dougherty, "Fuzzy mathematical morphology”, J. Visual Communication and Image Representation, vol. 3, no. 3, pp. 286-302, 1992.

[12] L. A. Zadeh, "Fuzzy Sets", Information and Control, vol. 8, pp. 338-353, 1965. 

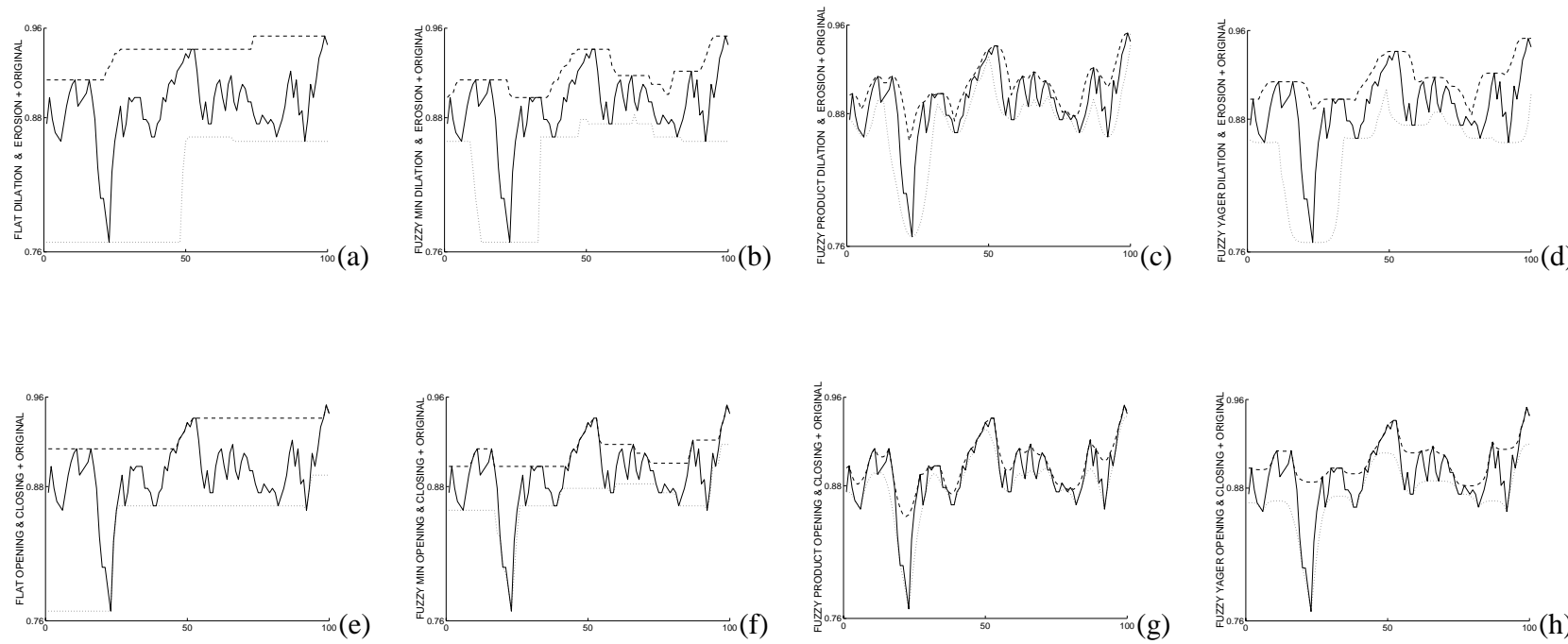

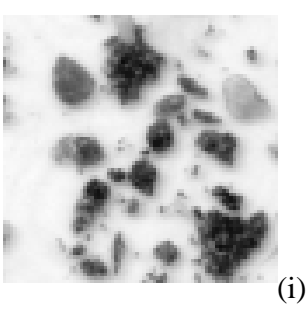

(i)

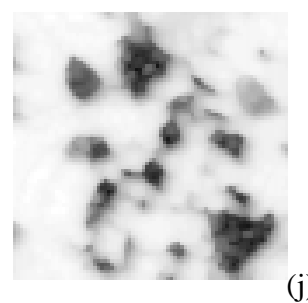

(j)
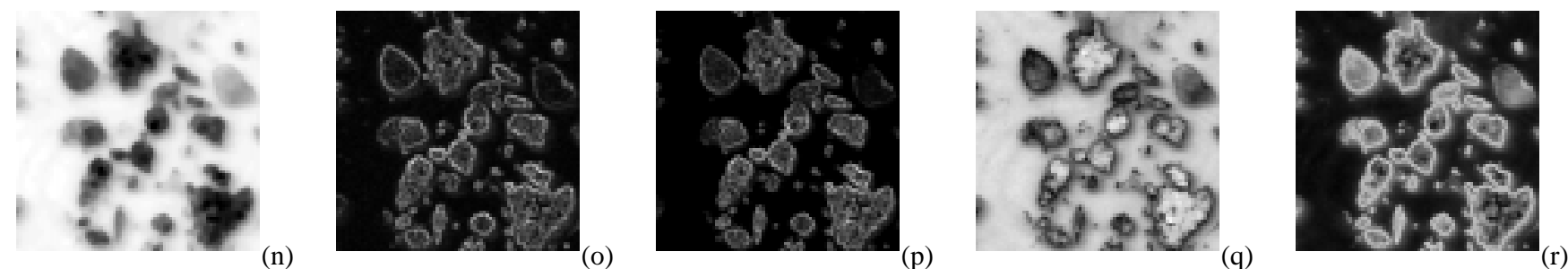
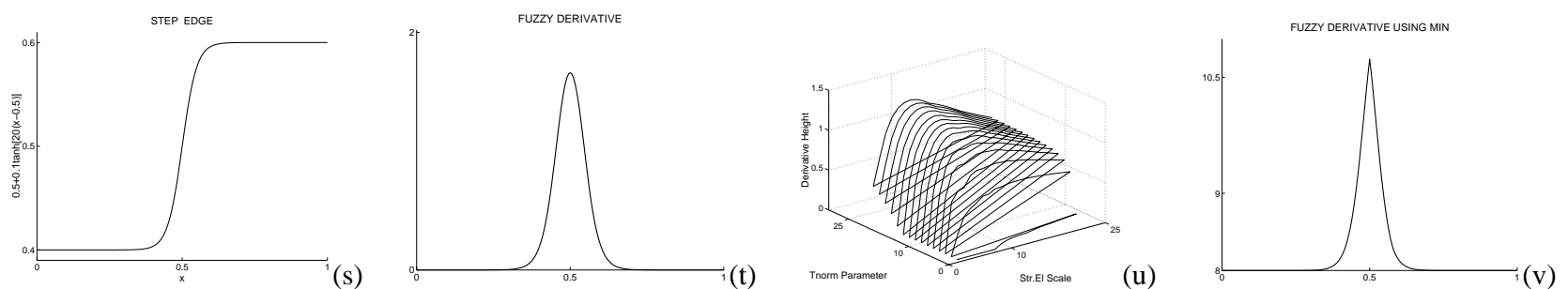

Fig. 1. Rows 1 and 2, left to right: flat, minimum, product, Yager. Row 1: original signal (solid line), dilation (dashed line), erosion (dotted line). Row 2: closing (dashed line), opening (dotted line). Row 3: (i) original image, (j) morphological dilation, (k) morphological erosion, (l) fuzzy dilation, (m) fuzzy erosion Row 4: (n) clos-opening, (o) morphological gradient, (p) fuzzy gradient $\delta-\varepsilon$, (q) fuzzy gradient $\min (\delta, 1-\varepsilon)$, (r) fuzzy gradient $\max (\delta, 1-\varepsilon)$. Row 5: (s) original step-edge signal $F$, (t) fuzzy derivative $[\delta(F)-\varepsilon(F)] /(2 s \Delta x)$, (u) 3D graph showing the heights of the derivatives (t) as the scale $s$ and parameter $p$ vary, (v) fuzzy derivative $[\min (\delta(F), 1-\varepsilon(F))] /(2 s \Delta x)$. 